\title{
Glutathione-S-Transferase P1 Isoenzyme Polymorphisms, Platinum-Based Chemotherapy, and Non-small Cell Lung Cancer
}

\author{
Richard Booten, MB ChB, MRCP, *† Tim Ward, PhD, † Jim Heighway, PhD, \& Linda Ashcroft, BSc, * \\ Julie Morris, PhD, $\neq$, and Nicholas Thatcher, PhD, FRCP* $\neq$
}

\begin{abstract}
Background: Polymorphisms within the P1 isoenzyme of GST (GSTP1) are associated with alterations in enzyme activity and may change sensitivity to platinum-based chemotherapy. We investigated the relationship between exon 5 and exon 6 GSTP1 gene polymorphisms and treatment response, hematological, and nonhematological toxicity and overall survival for patients receiving platinumbased chemotherapy for advanced non-small cell lung cancer (NSCLC).
\end{abstract}

Methods: Between 2001 and 2002, 108 patients with chemotherapy-naïve advanced NSCLC were recruited. Associations between the GSTP1 polymorphisms (Ile105Val, Thr110Ser, Ala114Val, and Asp 147Tyr) and GSTP1*A, *B, and ${ }^{*} \mathrm{C}$ haplotypes and treatment response and toxicity were evaluated using the Pearson $\chi^{2}$ and Kruskal-Wallis tests, respectively. Associations with survival were compared using Kaplan-Meier survival curves and Cox proportional hazard ratios.

Results: No significant associations were noted between GSTP1 polymorphisms and treatment response or survival. Significantly less neutropenic toxicity was demonstrated for patients possessing the $105 \mathrm{Val}$ allele $(p=0.020)$ or the GSTP $1 * \mathrm{~B}$ haplotype $(p=$ 0.038). However, the variant allele GSTP1 105Val, and patients possessing a GSTP $1 * \mathrm{~B}$ allele demonstrated notable trends toward inferior response and survival.

Conclusions: GSTP1 haplotype can be used to stratify hematological toxicity after platinum-based chemotherapy, but the lack of significant associations with response or survival suggests that GSTP1 polymorphisms may not be strong pharmacogenomic markers in this population. Additional large prospective studies incorporating the GSTP1 haplotype may clarify the reported discrepancies.

Key Words: GSTP1, Polymorphisms, Pharmacogenomics, Nonsmall cell lung cancer.

(J Thorac Oncol. 2006;1: 679-683)

\footnotetext{
*Christie Hospital NHS Trust, $\uparrow$ Paterson Institute for Cancer Research, and ISouth Manchester University Hospital NHS Trust, Manchester, and $\S$ Roy Castle International Centre for Lung Cancer Research, Liverpool, United Kingdom.

Address for correspondence: Richard Booten, MB ChB, MRCP, 28 Wrenbury Drive, Rochdale, Lancs, UK OL16 4PJ. E-mail: r.booten@btopenworld.com Copyright (C) 2006 by the International Association for the Study of Lung Cancer

ISSN: $1556-0864 / 06 / 0107-0679$
}

W orldwide, lung cancer is responsible for more than one million deaths per annum and is the leading cause of cancer mortality. ${ }^{1}$ Palliative treatments for locally advanced and metastatic non-small cell lung cancer (NSCLC), with chemotherapy and/or radiotherapy, have become a standard of care in this group but a therapeutic plateau has been reached..$^{2-4}$ In an attempt to advance the utility of current regimens, a pharmacogenetic approach has been advocated. 5,6 This concept "seeks to reduce the variation in how people respond to medicines by tailoring therapy to individual genetic make-up." 5 The identification of genetic markers that are able to stratify patients more or less likely to benefit from treatment may help to develop alternative strategies to improve the outcomes of anticancer chemotherapy.

Platinum-based chemotherapy is the mainstay of NSCLC treatment. Unfortunately, as many as $40 \%$ of patients do not respond to first-line platinum-based chemotherapy, ${ }^{4}$ although much is known about the potential mechanisms of platinum resistance. ${ }^{7-9}$ One such mechanism includes the inactivation of intracellular cisplatin by glutathione.

Increased intracellular glutathione levels is associated with previous exposure to platinum drugs and subsequent platinum resistance, ${ }^{10-13}$ while down-regulating glutathionerelated enzymes or glutathione levels may improve cisplatin sensitivity, 12,14-17 by allowing aquated platinum species to react with intracellular target molecules such that potentially cytotoxic cisplatin-DNA monoadducts may convert to intrastrand crosslinks. ${ }^{18}$ Increased expression of glutathione-S-transferase P1 isoenzyme (GSTP1), one of five glutathione-S-transferase isoenzymes, has been demonstrated to increase intracellular glutathione and increase the proportion of GSH-Pt adduct ${ }^{19}$ and to alter the response of patients undergoing platinum-based chemotherapy in a variety of solid tumors. ${ }^{20-24}$

Consequently, we sought to identify whether nonsynonymous polymorphisms at a number of loci within GSTP1 affected toxicity or response to chemotherapy and survival in patients with advanced NSCLC receiving platinum-based chemotherapy as part of a phase III randomized trial.

\section{PATIENTS AND METHODS}

\section{Patient Selection}

Between June 2001 and November 2002, 433 patients participated in a randomized phase III trial of docetaxel/ 
TABLE 1. Polymerase Chain Reaction and Sequencing Primers for XPD and GSTP1 Polymorphisms

\begin{tabular}{|c|c|c|c|c|c|}
\hline & Polymorphism & & & Primer sequences & Tm \\
\hline \multirow[t]{4}{*}{ GSTP1 } & Ile105Val & \multirow[t]{2}{*}{ Exon5 } & $\mathrm{F} / \mathrm{S}$ & TGATCAGGCGCCCAGTCA & \multirow[t]{2}{*}{52} \\
\hline & Thr110Ser & & $\mathrm{R}$ & AAGAAGCCCCTTTCTTTGTTC & \\
\hline & Ala114Val & \multirow[t]{2}{*}{ Exon6 } & $\mathrm{F} / \mathrm{S}$ & GAGCAAGCAGAGGAGAATCTG & \multirow[t]{2}{*}{57} \\
\hline & Asp147Tyr & & $\mathrm{R}$ & ACAAATGGCTCACACCTGTGT & \\
\hline
\end{tabular}

$\mathrm{F}, 5^{\prime}$ to $3^{\prime}$ primer; $\mathrm{R}, 3^{\prime}$ to $5^{\prime}$ primer, $\mathrm{S}$, sequencing primer.

carboplatin versus mitomycin, ifosfamide, cisplatin (MIC) or mitomycin, vinblastine, cisplatin (MVP) in advanced NSCLC from 16 institutions across the United Kingdom. Patients recruited from two hospitals in South Manchester, UK (Wythenshawe and Christie Hospitals) provided additional informed consent for genotyping from whole blood. Patients consenting to this represent the genotype cohort reported herein. The local research and ethics committee approved the study. The age, stage of disease, histology, and Eastern Cooperative Group (ECOG) performance status were obtained at study entry. Follow-up information, treatment response, and survival were derived from the clinical trials database at the Christie Hospital.

All patients enrolled in the study had pathologically confirmed stage III-IV chemotherapy-naïve NSCLC with an ECOG performance status of 0-2 and received either docetaxel $75 \mathrm{mg} / \mathrm{m}^{2}$ and carboplatin AUC 6 every 3 weeks or mitomycin C $6 \mathrm{mg} / \mathrm{m}^{2}$, cisplatin $50 \mathrm{mg} / \mathrm{m}^{2}$, and vinblastine 6 $\mathrm{mg} / \mathrm{m}^{2}$ or ifosfamide $3 \mathrm{~g} / \mathrm{m}^{2}$ every 3 weeks to a maximum of four cycles. Tumors were required to be measurable in at least one dimension using the Response Evaluation Criteria in Solid Tumors. ${ }^{25}$ Briefly, responses were determined in relation to the change in the sum of long-axis dimensions (LD) for all target lesions between baseline and 4 weeks after completion of chemotherapy. A complete response (CR) was identified if no residual disease was evident, a partial response (PR) if there was $>30 \%$ reduction in the sum LD, progressive disease (PD) if $>20 \%$ increase in the sum LD, and stable disease (SD) if criteria not met for CR, PR, and PD. Responses were determined at the end of chemotherapy by trial investigators without previous knowledge of clinical outcome. Toxicity was assessed using the National Cancer Institutes Common Toxicity Criteria (Version 2.0, 1/30/98) and maximum toxicity recorded for each cycle of chemotherapy administered.

\section{DNA Extraction and Genotyping}

Peripheral blood was drawn following randomization and enrollment into the chemotherapy trial, and DNA was extracted from these samples using the QIAamp Blood DNA Midi Kit (Qiagen, Crawley, UK) and $2 \mathrm{ml}$ of whole blood according to manufacturer's instructions. The known nonsynonymous polymorphisms of exons 5 and 6 of GSTP1 (GSTP1 Ile105Val, Thr110Ser, Ala114Val, and Asp 147Tyr) were determined using two independent runs of polymerase chain reaction and direct sequencing followed by data analysis using PhredPhrap, PolyPhred, and Consed software packag- es. ${ }^{26-29}$ If both sequencing reactions were in agreement, the genotype was considered robust. Haplotypes were determined according to Ishimoto and Ali-Osman ${ }^{30}$ and were as follows: $* \mathrm{~A}=105 \mathrm{Ile} / 114 \mathrm{Ala},{ }^{*} \mathrm{~B}=105 \mathrm{Val} / 114 \mathrm{Ala},{ }^{*} \mathrm{C}=$ $105 \mathrm{Val} / 114 \mathrm{Val}$. Polymerase chain reaction oligonucleotides were obtained from MWG Biotech (Milton Keynes, UK) after a BLASTN search to ensure $100 \%$ sequence homology with the gene of interest (accession number GSTP1AY324387). Oligonucleotide sequences and annealing temperatures are listed in Table 1 and reactions performed for 38 polymerase chain reaction cycles.

\section{RESULTS}

\section{Trial Demographics and Genetic Frequencies}

Among the 179 patients who were randomized to chemotherapy, 108 patients provided whole blood for genotyping (genotype cohort). No statistically significant differences in age, sex, histology, stage, and performance status were demonstrated between the chemotherapy arms and therefore the genotype cohort was analyzed as a single group.

The genotype cohort included 74 (68.5\%) males and 34 (31.5\%) females with a median age 62.5 years (range, $35-80$ years). The median overall survival time was 282 days $(95 \%$ confidence interval [CI]: 221-343 days). Fifty-two percent $(55 / 108)$ of the study participants died of disease during the study period. Of the 108 patients, clinical response and GSTP1 genotype could be determined for $88(81 \%)$ and 90 $(83 \%)$ patients with exon 5 and exon 6 GSTP1 genotypes, respectively. The genotype and allele frequencies are listed in Table 2. The variant allele of the GSTP1 110 and 147

TABLE 2. Genotype Distributions of the GSTP1 Gene in Patients with Advanced Non-small Cell Lung Cancer

\begin{tabular}{lcccc}
\hline Genotype & \multicolumn{2}{c}{$\begin{array}{c}\text { Ile105Val } \\
\mathbf{A} \rightarrow \mathbf{G} \\
(\boldsymbol{n}=\mathbf{9 6})\end{array}$} & \multicolumn{2}{c}{$\begin{array}{c}\text { Ala114Val } \\
\mathbf{C} \rightarrow \mathbf{T} \\
(\boldsymbol{n}=\mathbf{9 8})\end{array}$} \\
\hline Wild type & $\mathrm{AA}$ & 44.8 & $\mathrm{CC}$ & 87.8 \\
Heterozygote & $\mathrm{AG}$ & 38.5 & $\mathrm{CT}$ & 12.2 \\
Homozygous variant & $\mathrm{GG}$ & 16.7 & $\mathrm{TT}$ & 0 \\
Allele frequency & $\mathrm{A}$ & 0.640 & $\mathrm{C}$ & 0.939 \\
& $\mathrm{G}$ & 0.360 & $\mathrm{~T}$ & 0.061 \\
\hline
\end{tabular}

Genotypes were found to be in Hardy-Weinberg equilibrium with no significant differences between observed and expected frequencies $\left(\chi^{2}\right.$ test, $\left.p>0.05\right)$. 
TABLE 3. GSTP1 Genotype and Tumor Response

\begin{tabular}{llcc}
\hline Genotype & ORR & SD & PD \\
\hline GSTP1 105 $(n=86, p=0.93)$ & & & \\
Wild-type Ile/Ile $(n=38)$ & 34.2 & 31.6 & 34.2 \\
Heterozygote Ile/Val $(n=32)$ & 37.5 & 28.1 & 34.4 \\
Variant Val/Val $(n=16)$ & 25 & 37.5 & 37.5 \\
GSTP1 114 $(n=89, p=0.54)$ & & & \\
Wild-type Ala/Ala $(n=77)$ & 32.5 & 33.8 & 33.8 \\
Heterozygote Ala/Val $(n=12)$ & 45.5 & 18.2 & 36.4 \\
Variant Val/Val $(n=0)$ & 0 & 0 & 0 \\
GSTP1 haplotypes $(p=0.82)$ & & & \\
Ile105Ile/Ala114Ala AA $(n=38)$ & 32.0 & 31.6 & 34.2 \\
Ile105Val/Ala114Ala AB $(n=25)$ & 28.6 & 36.0 & 32.0 \\
Val105Val/Ala114Ala BB $(n=13)$ & 23.1 & 38.5 & 38.5 \\
Ile105Val/Ala114Val AC $(n=7)$ & 57.1 & 0 & 42.9 \\
Val105Val/Ala114Val BC $(n=3)$ & 33.3 & 33.3 & 33.3 \\
GSTP1 by B haplotype $(p=0.56)$ & & & \\
B haplotypes $(n=41)$ & 29.3 & 36.6 & 34.1 \\
Non-B haplotypes $(n=45)$ & 37.8 & 26.7 & 35.6 \\
\hline
\end{tabular}

GSTP1 haplotypes: *A, 105Ile/114Ala; *B, 105Val/114Ala; *C, 105Val/114Val. ORR, objective response rate; $\mathrm{SD}$, stable disease; $\mathrm{PD}$, progressive disease.

polymorphisms were not detected in this patient group and were not subject to further evaluation.

\section{Tumor Response}

For patients receiving chemotherapy, there was no statistically significant difference in response to chemotherapy between the two arms of chemotherapy. Consequently, an objective response was documented in $30.5 \%$, stable disease in $28.2 \%$ and progressive disease in $29.2 \%$.

No significant relationships were demonstrated between the GSTP1 105 or 114 variant allele according to response to platinum-based chemotherapy ( $p=0.93$ and $p=$ 0.54 , respectively). Similarly, response to chemotherapy was not significantly associated with GSTP1 haplotype $(p=0.82)$ (Table 3). However, patients possessing one or more * $\mathrm{B}$ allele exhibited an inferior tumor response compared to patients who did not possess a *B allele.

\section{Survival}

No statistically significant differences in survival were seen between the two chemotherapy arms in the genotype cohort. Stage of disease was significantly associated with survival ( $p=0.024$ ) but age, histology, performance status, and sex demonstrated no association.

For GSTP1 genotypes (Table 4), no significant difference in median survival was demonstrated for the GSTP1 105 or 114 polymorphic loci. However, the variant exon 6 genotype demonstrated greater median survival (Figure 1) and was associated with a reduction in hazard ratio of death of 0.56 .

GSTP1 haplotype demonstrated no significant relationship with survival after platinum-based chemotherapy, but possession of one or more GSTP $1 * \mathrm{~B}$ allele was associated with a nonsignificant $22 \%$ increased risk of death.

\section{Toxicity}

No significant difference in grade of toxicity for hemoglobin level or total white cell count was evident for GSTP1 genotype/haplotype. However, significant differences in grade of neutropenia were seen for patients according to GSTP1 105 and GSTP1 haplotypes such that the rate of high-grade neutropenia was significantly decreased by the 105 variant allele and by the GSTP1*B allele (Table 5).

For nonhematological toxicity, no significant associations with the GSTP1 114 allele were demonstrated, although for GSTP1 105, a significantly greater proportion of patients

TABLE 4. GSTP1 Genotype and Survival

\begin{tabular}{|c|c|c|c|c|c|}
\hline & Median survival (mo) & $95 \% \mathrm{CI}$ & $p$ & HR & $95 \% \mathrm{CI}$ \\
\hline Genotype cohort & 9.3 & $7.3-11.3$ & & & \\
\hline \multicolumn{6}{|l|}{ GSTP1 105} \\
\hline Wild type & 9.3 & $7.5-11.0$ & 0.72 & 1.0 & \\
\hline Heterozygote & 10.8 & $5.6-15.9$ & & 0.83 & $0.44-1.58$ \\
\hline Variant & 7.7 & $6.5-8.9$ & & 1.14 & $0.52-2.50$ \\
\hline \multicolumn{6}{|l|}{ GSTP1 114} \\
\hline Wild type & 9.2 & $7.0-11.3$ & 0.18 & 1.0 & \\
\hline Heterozygote & 11.9 & $8.6-15.1$ & & 0.56 & $0.24-1.33$ \\
\hline Variant & - & - & & - & - \\
\hline \multicolumn{6}{|l|}{ GSTP1 haplotypes } \\
\hline Ile105Ile/Ala114Ala AA $(n=38)$ & 9.3 & $7.5-11.0$ & 0.70 & & \\
\hline Ile105Val/Ala114Ala AB $(n=25)$ & 8.0 & $5.5-10.5$ & & & \\
\hline Val105Val/Ala114Ala BB $(n=13)$ & 8.1 & $1.6-14.6$ & & & \\
\hline Ile105Val/Ala114Val AC $(n=7)$ & 11.9 & $2.6-21.2$ & & & \\
\hline Val105Val/Ala114Val BC $(n=3)$ & - & - & & & \\
\hline $\mathrm{B}$ haplotypes (AB, BB, BC) & 8.0 & $6.7-9.3$ & 0.49 & 1.22 & $0.69-2.13$ \\
\hline Non-B haplotypes (AA, AC) & 10.8 & $8.6-12.8$ & & 1.0 & \\
\hline
\end{tabular}




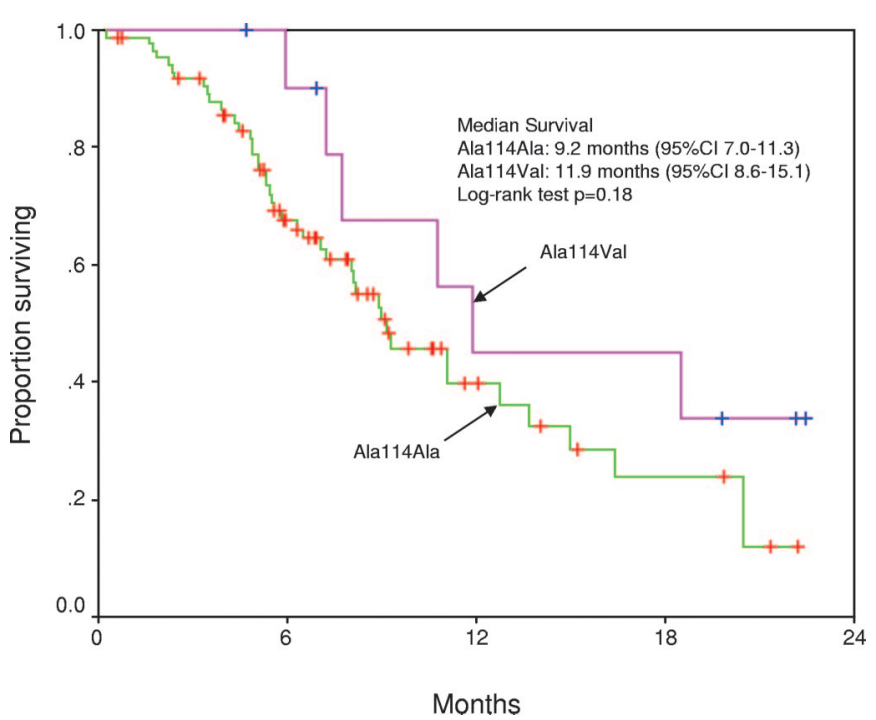

FIGURE 1. Kaplan-Meier survival curves for patients receiving platinum-based chemotherapy by GSTP1 114 genotype. $\mathrm{Cl}$, confidence interval.

of homozygous variant genotype possessed grade 0 mucositis compared to the other genotypes $(p=0.021)$.

\section{DISCUSSION}

In view of the current therapeutic plateau in treatment for advanced NSCLC, we sought to evaluate whether nonsynonymous polymorphisms in GSTP1 were associated with toxicity or response to chemotherapy or influenced survival after platinum-based chemotherapy.

GSTs play an important role in detoxification by catalyzing the conjugation of many compounds with reduced glutathione. Four main classes exist and include the GSTP1 isoenzyme that is expressed at particularly high levels in normal lung tissue, in lung cancer, and in malignant tissue relative to matched normal tissues. ${ }^{31}$ Four nonsynonymous polymorphic variants are reported for GSTP1 (http:// www.ncbi.nlm.nih.gov/SNP/snp_ref.cgi?locusId=2950), although in our population of patients with advanced NSCLC, only the codon 105 and 114 polymorphisms were present. In 1998, Watson et al. ${ }^{32}$ considered these polymorphisms in surgically resected normal lung tissue and their effect on the glutathione-conjugating ability of GSTP1. Significant differences in conjugating ability were found for Ile105Val, with wild-type 105Ile genotypes being most protective and variant $105 \mathrm{Val}$ genotypes the least protective $(p<0.05)$. However, no relationship for the Ala1 $14 \mathrm{Val}$ polymorphism and altered enzyme activity was elicited. In vivo data are in keeping with this observation, as patients who possess the $105 \mathrm{Val}$ variant demonstrate elevated DNA adducts (45\% higher) in normal breast tissue compared to wild-type genotypes. ${ }^{33}$ Further supportive data come from Stoehlmacher et al.,34,35 in a study of 106 colorectal cancer patients receiving oxaliplatin, who noted significantly improved survival for variant genotypes (Val105Val) compared to wild-type patients. However, significance was lost after stratification for performance status. Furthermore, in a study of more than 400 patients with advanced NSCLC, Lu et al. ${ }^{36}$ demonstrated that variant exon 6 genotypes (114Val), but not variant exon 5 genotypes (105Val), were associated with increased survival and a reduced adjusted hazard ratio for death of 0.75 . Our results did not produce statistically significant results for the GSTP1 105 or 114 loci according to response or survival data but are in keeping with these observations. We have also extended these observations to include an analysis of GSTP1 haplotypes that have variable cytoprotective activities, GSTP1*A being least protective and GSTP $1 * \mathrm{C}$ most protective in response to platinum drug administration. ${ }^{30}$ GSTP $1 * \mathrm{~A}$ is the most common allele in our population (0.628), with allele

TABLE 5. Common Toxicity Criteria for Neutropenia, GSTP1 Polymorphisms, and Platinum-Based Chemotherapy

\section{CTC Grade for Neutropenia}

\begin{tabular}{|c|c|c|c|c|c|c|}
\hline \multirow[b]{2}{*}{ Genotype } & \multicolumn{6}{|c|}{ CTC Grade for Neutropenia } \\
\hline & $\mathbf{0}$ & 1 & 2 & 3 & 4 & $p$ \\
\hline \multicolumn{7}{|l|}{ GSTP1 $105(n=93)$} \\
\hline Wild type & 14.6 & 12.2 & 0 & 7.3 & 65.9 & 0.020 \\
\hline Heterozygote & 5.4 & 2.7 & 8.1 & 21.6 & 62.2 & \\
\hline Variant & 6.7 & 6.7 & 26.7 & 13.3 & 46.7 & \\
\hline \multicolumn{7}{|l|}{ GSTP1 $114(n=95)$} \\
\hline Wild type & 9.5 & 7.1 & 7.1 & 13.3 & 63.1 & 0.98 \\
\hline Heterozygote/variant & 9.1 & 9.1 & 9.1 & 18.2 & 54.5 & \\
\hline \multicolumn{7}{|c|}{ GSTP1 haplotype $(n=93)$} \\
\hline AA & 14.6 & 12.2 & 0 & 7.3 & 65.9 & 0.015 \\
\hline $\mathrm{AB}$ & 3.4 & 3.4 & 6.9 & 24.1 & 62.1 & \\
\hline $\mathrm{AC}$ & 12.5 & 0 & 12.5 & 12.5 & 62.5 & \\
\hline BB & 7.7 & 0 & 30.8 & 7.7 & 53.8 & \\
\hline $\mathrm{BC}$ & 0 & 50 & 0 & 50 & 0 & \\
\hline $\mathrm{AB}, \mathrm{BB}, \mathrm{BC}$ & 4.5 & 4.5 & 13.6 & 20.6 & 56.8 & 0.038 \\
\hline $\mathrm{AA}, \mathrm{AC}$ & 14.3 & 10.2 & 2.0 & 8.2 & 65.3 & \\
\hline
\end{tabular}


frequencies for GSTP1*B and C of 0.314 and 0.058 , respectively. Our data suggest that the GSTP $1 * \mathrm{~B}$ allele is associated with a reduced response to platinum drugs and a poorer survival and are in keeping with the greater protective activity reported in vitro compared to GSTP1*A. In addition, and further supporting the protective role of the GSTP $1 * \mathrm{~B}$ haplotype, are the significant differences in hematological toxicity demonstrated between patients who possess at least one copy of the GSTP1*B allele compared to patients without.

This report represents one of the largest series in advanced NSCLC evaluating a pharmacogenomic approach to therapy. Polymorphisms in GSTP1 do not appear to be strong biomarkers for the predictive prescription of platinumbased chemotherapy, but our data suggest that differential GSTP1 enzyme activity can be identified by GSTP1 haplotype but requires confirmation in a larger sample size. Welldesigned prospective, randomized clinical trials using these biomarkers should clarify the reported discrepancies between the published studies and delineate their place in clinical practice.

\section{REFERENCES}

1. Shibuya K, Mathers CD, Boschi-Pinto C, Lopez AD, Murray CJ. Global and regional estimates of cancer mortality and incidence by site: II. Results for the global burden of disease 2000. BMC Cancer 2002;2:37.

2. Kelly K, Crowley J, Bunn PA Jr, et al. Randomized phase III trial of paclitaxel plus carboplatin versus vinorelbine plus cisplatin in the treatment of patients with advanced non-small-cell lung cancer: a Southwest Oncology Group trial. J Clin Oncol 2001;19:3210-3218.

3. Scagliotti GV, De Marinis F, Rinaldi M, et al. Phase III randomized trial comparing three platinum-based doublets in advanced non-small-cell lung cancer. J Clin Oncol 2002;20:4285-4291.

4. Schiller JH, Harrington D, Belani CP, et al. Comparison of four chemotherapy regimens for advanced non-small-cell lung cancer. $N$ Engl $J$ Med 2002;346:92-98

5. Goldstein DB, Tate SK, Sisodiya SM. Pharmacogenetics goes genomic. Nat Rev Genet 2003;4:937-947.

6. Marsh S, McLeod HL. Cancer pharmacogenetics. Br J Cancer 2004;90: $8-11$.

7. Brabec V, Kasparkova J. Molecular aspects of resistance to antitumor platinum drugs. Drug Resist Update 2002;5:147-161.

8. Kartalou M, Essigmann JM. Mechanisms of resistance to cisplatin. Mutat Res 2001;478:23-43.

9. Kartalou M, Essigmann JM. Recognition of cisplatin adducts by cellular proteins. Mutat Res 2001;478:1-21.

10. Newkirk K, Heffern J, Sloman-Moll E, et al. Glutathione content but not gamma glutamyl cysteine synthetase mRNA expression predicts cisplatin resistance in head and neck cancer cell lines. Cancer Chemother Pharmacol 1997:40:75-80.

11. Juvekar AS, Adwankar MK, Tongaonkar HB. Effect of cisplatin-based chemotherapy on emergence of cisplatin resistance, and its correlation with intracellular glutathione levels and accumulation of $\mathrm{p} 53$ protein in human ovarian cancer. Cancer Biother Radiopharm 2000;15:295-300.

12. Ikeda K, Miura K, Himeno S, Imura N, Naganuma A. Glutathione content is correlated with the sensitivity of lines of PC12 cells to cisplatin without a corresponding change in the accumulation of platinum. Mol Cell Biochem 2001;219:51-56.

13. Lewandowicz GM, Britt P, Elgie AW, et al. Cellular glutathione content, in vitro chemoresponse, and the effect of BSO modulation in samples derived from patients with advanced ovarian cancer. Gynecol Oncol 2002;85:298-304.

14. Ogawa J, Iwazaki M, Inoue $\mathrm{H}$, Koide S, Shohtsu A. Immunohistochemical study of glutathione-related enzymes and proliferative antigens in lung cancer. Relation to cisplatin sensitivity. Cancer 1993;71:22042209.

15. Ban N, Takahashi Y, Takayama T, et al. Transfection of glutathione
S-transferase (GST)-pi antisense complementary DNA increases the sensitivity of a colon cancer cell line to Adriamycin, cisplatin, melphalan, and etoposide. Cancer Res 1996;56:3577-3582.

16. Nagata J, Kijima H, Hatanaka H, et al. Reversal of cisplatin and multidrug resistance by ribozyme-mediated glutathione suppression. Biochem Biophys Res Commun 2001;286:406-413.

17. Goto S, Kamada K, Soh Y, Ihara Y, Kondo T. Significance of Nuclear Glutathione S-Transferase pi in Resistance to Anti-cancer Drugs. Jpn J Cancer Res. 2002;93:1047-56.

18. Eastman A. Cross-linking of glutathione to DNA by cancer chemotherapeutic platinum coordination complexes. Chem Biol Interact 1987;61: 241-248.

19. Goto S, Iida T, Cho S, et al. Overexpression of glutathione S-transferase pi enhances the adduct formation of cisplatin with glutathione in human cancer cells. Free Radic Res 1999;31:549-558.

20. Hamada S, Kamada K, Furumoto H, Hirao T, Aono T. Expression of glutathione S-transferase-pi in human ovarian cancer as an indicator of resistance to chemotherapy. Gynecol Oncol 1994;52:313-319.

21. Nishimura T, Newkirk K, Sessions RB, et al. Immunohistochemical staining for glutathione S-transferase predicts response to platinumbased chemotherapy in head and neck cancer. Clin Cancer Res 1996;2: 1859-1865.

22. Bai F, Nakanishi Y, Kawasaki M, et al. Immunohistochemical expression of glutathione S-transferase-Pi can predict chemotherapy response in patients with nonsmall cell lung carcinoma. Cancer 1996;78:416-421.

23. Li L, Yao D, Chen X. [The expression of glutathione S-transferase pi in human ovarian cancer as an indicator of resistance to chemotherapy]. Zhonghua Fu Chan Ke Za Zhi 1998;33:95-97.

24. Arai T, Yasuda Y, Takaya T, et al. Immunohistochemical expression of glutathione transferase-pi in untreated primary non-small-cell lung cancer. Cancer Detect Prev 2000;24:252-257.

25. Therasse P, Arbuck SG, Eisenhauer EA, et al. New Guidelines to Evaluate the Response to Treatment in Solid Tumors. J Natl Cancer Inst 2000;92:205-216.

26. Nickerson DA, Tobe VO, Taylor SL. PolyPhred: automating the detection and genotyping of single nucleotide substitutions using fluorescence-based resequencing. Nucleic Acids Res 1997;25:2745-2751.

27. Gordon D, Abajian C, Green P. Consed: a graphical tool for sequence finishing. Genome Res 1998;8:195-202.

28. Ewing B, Hillier L, Wendl MC, Green P. Base-calling of automated sequencer traces using Phred. I. Accuracy assessment. Genome Res 1998;8:175-185.

29. Ewing B, Green P. Base-calling of automated sequencer traces using Phred. II. Error probabilities. Genome Res 1998;8:186-194.

30. Ishimoto TM, Ali-Osman F. Allelic variants of the human glutathione S-transferase P1 gene confer differential cytoprotection against anticancer agents in Escherichia coli. Pharmacogenetics 2002;12:543-553.

31. Moscow JA, Fairchild CR, Madden MJ, et al. Expression of anionic glutathione-S-transferase and P-glycoprotein genes in human tissues and tumors. Cancer Res 1989;49:1422-1428.

32. Watson MA, Stewart RK, Smith GB, Massey TE, Bell DA. Human glutathione S-transferase P1 polymorphisms: relationship to lung tissue enzyme activity and population frequency distribution. Carcinogenesis. 1998; 19:275-280.

33. Brockstedt U, Krajinovic M, Richer C, et al. Analyses of bulky DNA adduct levels in human breast tissue and genetic polymorphisms of cytochromes P450 (CYPs), myeloperoxidase (MPO), quinone oxidoreductase (NQO1), and glutathione S-transferases (GSTs). Mutat Res 2002;516:41-47.

34. Stoehlmacher J, Park DJ, Zhang W, et al. Association between glutathione S-transferase P1, T1, and M1 genetic polymorphism and survival of patients with metastatic colorectal cancer. J Natl Cancer Inst 2002; 94:936-942.

35. Stoehlmacher J, Park DJ, Zhang W, et al. A multivariate analysis of genomic polymorphisms: prediction of clinical outcome to 5-FU/oxaliplatin combination chemotherapy in refractory colorectal cancer. $\mathrm{Br} J$ Cancer 2004;91:344-54.

36. Lu C, Spitz MR, Zhao $\mathrm{H}$, et al. Association between glutathione S-transferase pi polymorphisms and survival in patients with advanced nonsmall cell lung carcinoma. Cancer 2006;106:441-447. 